\title{
The Importance of Reducing the Viral Load in the Oral Cavity
}

\author{
Michelle Moron Araújo ${ }^{1,2^{*}}$
}

${ }^{1}$ Dentist, Pontificia Universidad Javeriana Bogotá, Colombia

${ }^{2}$ Specialist in Periodontology, Pontificia Universidad Católica Buenos Aires, Argentina

*Corresponding author: Michelle Moron Araújo, Dentist, Pontificia Universidad Javeriana Bogotá, Transv19A \#97-59 Bogotá, Colombia; Specialist in Periodontology, Pontificia Universidad Católica Buenos Aires, Argentina

\begin{abstract}
Coronavirus infection has been considered by the WHO (World Health Organization) as a global pandemic. Since its origin, it has posed a threat to global health, not so much because of its symptoms, but because of its rapid spread, contagion and the respiratory complications that it produces in patients with other chronic underlying conditions. The oral cavity has a high viral load, which is very important for oral hygiene and all biosafety protocols.
\end{abstract}

\section{Introduction}

In this year 2020, the world population has been affected by the COVID-19 coronavirus that originated in the city of Wuhan in China in the last month of the year 2019. This pandemic has brought negative health, economic, social, etc. repercussions [1].

We have learned throughout these months that this virus is an invisible enemy, powerful and capable of causing much damage, observing the high number of infections and high fatality rates worldwide. To help to combat this pandemic, the educational and awareness-raising part about general and oral hygiene is very important such as hand washing and tooth brushing.

In the stomatognathic system, including the oral cavity, it plays an important role since it is one of the main entry routes for viruses, bacteria, fungi, microorganisms that can affect the general health of an individual. The scientific evidence of COVID-19 with the oral cavity is extensive and the role of the oral cavity and saliva [2].

The new coronavirus increases the contagion capacity of ACE 2 receptors with high expression in oral tissues such as tongue, oral mucosa, gingival tissues, indicating that the oral cavity is a potential risk route for COVID-19 infection. This viral load means the ability of the virus to increase the number of replications and to damage. This viral load can last 3 or 4 weeks, the incubation period lasts approximately 7 days and viral samples lasts up to 60 days [3].

This estimate of the amount of viral particles in body fluids can be studied in saliva, blood, eses and urine. When the virus enters the body, it invades cells and replicates the number of copies throughout the days. The viral load in the mouth is high, so the oropharynx swab test is the most reliable $[4,5]$.

If a person has more viral particles each day in his body, the disease will be more severe and the possibility of contagion will be higher. That is why people, who are exposed to a high viral load, are those who are more likely to have a more severe disease. Viral load is an important factor with the prognosis of the patient and helps to manage and control the virus [3].

A cross infection can occur in members of a family or people who live together if the corresponding biosecurity standards are not maintained. Drops of saliva are a way of contagion of COVID-19 which infected people will present different symptoms such as: Fatigue, muscle pain, fever, loss of taste and smell. Oral manifestations of COVID-19 have been reported, such as ulcers, petechiae, sialadenitis, enanthem, among others [4].

To reduce or mitigate the viral load in the mouth, physical distancing, the use of face masks, eye protection, hand washing, promoting and encouraging oral hygiene is important. The work of the dentist is very important since the oral cavity has a viral load and bac- 
terial load. COVID-19 creates conditions so that super infections by oral bacteria can occur in crevicular fluid and periodontium, as in the cases of patients with cardiovascular problems, diabetics, pneumonia, etc [5].

Poor oral hygiene and problems such as periodontitis, gingivitis, cavities, abscesses create appropriate environments for COVID-19 to have a severe picture. Lowering the viral load will reduce the repercussions at the systemic level and oral manifestations. Likewise, good oral hygiene reduces the morbidity and severity of COVID-19 [6].

To lower the oral viral and bacterial load, the work of the dentist and preventive measures in oral health are important, such as oral hygiene habits at home, teaching and applying correct brushing of teeth, gums and tongue, the use of dental floss, mouthwashes, comprehensive treatment and dental controls. The implications in clinical practice with preoperative mouthwashes significantly reduce the number of microorganisms in the aerosol in the dental office.

\section{Financing}

None.

\section{Conflict of Interest}

None.

\section{References}

1. Rabi FA, Al Zoubi MS, Kasasbeh GA, Salameh DM, Al-Nasser AD (2020) SARS-Cov-2 and Coronavirus disease 2019: What we know so far. Pathogens 9: 231.

2. Guan W, Ni Z, Hu Y, Liang W, Ou C, et al. (2020) Clinical characteristics of 2019 novel coronavirus infection in China. N Engl J Med.

3. Xu H, Zhong L, Deng J, Peng J, Dan H, et al. (2020) High expression of ACE2 receptor of 2019-nCoV on the epithelial cells of oral mucosa. Int J Oral Sci 12: 1-5.

4. Reza H, Omid S, Zandian D, Gon S, Cheshm B (2020) Being a front-line dentist during the covid-19 pandemic: A literature review. Maxillofac Plast Reconstr Surg 42: 12.

5. Carreras M, Sánchez A, López A, Salas J, Perez S (2020) Oral vesiculobullous lesions associated with SARS-CoV-2 infection. Oral Dis.

6. Shufa Z, Jian F, Fei Y, Baihuan F, Bin L, et al. (2020) Viral load dynamics and disease severity in patients infected with SARS-CoV-2 in Zhejiang province, China 2020: Retrospective cohort study. BMJ 369: 1-8. 